\title{
INFRARED IMAGINATIONS AND CLOUD-TRUTH Classifying Weather In The Satellite Age
}

By Charlotte Scott

T ike sex, weather is a social creation. Indeed, we talk about the Lweather more often than we talk about sex, and, as for most natural processes, society has created structures of meaning that define atmospheric change in human terms. These meanings evolve as technology and scientific thought progress, creating new ways of experiencing the weather and of literally seeing the sky. In modern, enlightened times, the weather has become subject to thorough classification, formulation and social regulation via new techniques of atmospheric observation and scientific processing. As the technocultural eye sees the atmosphere differently, ideas about what the weather means also change. Berland notes that the "most brazenly unruled of all the cyclical processes of 'Nature' turns out to be shaped differently by our different imaginations, and now haunts our material symbolic expressions through inversion, distortion, condensation, and absence" (1999). The endless sky becomes an endless series of digitized patterns and formulas, whose earthly results nonetheless connect to the most visceral and emotional centres of human consciousness.

There was a time in my life when I did not pay attention to weather reports. Living in the congenial wilds of the Rocky Mountains, it was far more convenient to stick my head out the door in the morning, assess the character of the air, and go from there. At the time I felt I had a more direct relationship with the atmosphere. Now, I check the weather obsessively, twice, three times a day. Not only do I check forecasts in my own region (which is all I really need to know from a functional standpoint), I consult a host of global satellite and radar images. I scan the weather in the prairies when I feel homesick; I even study the ice thickness and prevalence of icebergs 
off the western coast of Ellesmere Island when I feel adventurous. I consistently consult an array of pressure, humidity, dew point and precipitation readings, each measurement dedicated to one facet of the atmosphere's overall character for the day.

Needless to say, this information is not gathered by sticking my head out the door. Rather, one logs on to the inexhaustibly informative Environment Canada website and takes a virtual trip into space. , Currents of water vapour swirl about "below", invisible to the naked eye, and coloured infrared clouds hover in the sky like elegant whales twisting and banking in the jet stream. Enchanted by these digital projections, the observer has not once looked out the window. Indeed, the computer screen is the new porthole, enframing impossible visions of the Earth's upper atmosphere. As such, our "view from space" is mediated through a number of technological and cultural filters. These swirling clouds that one "sees" so clearly represented on the computer screen are actually fluctuating webs of raw data and vectors, reconstituted for our personal enjoyment, but also as a functional tool for industry, observation, defense, and research.

My understanding of the weather has evolved tremendously since I started hanging out in space with my satellite friends, GOES10 and GOES-12. These Geosynchronous Operational Environmental Satellites hover about 36,000km above the Earth's surface. They are designed to gather and transmit visual data and infrared images of the atmosphere above North and South America. Data is transmitted to stations on the ground, where it is examined, processed and reassembled into a product ready for consumption and analysis by any number of government, commercial and scientific organizations. Data is also obviously constructed for aesthetic pleasure, as evidenced by the numerous visualization and colour-coding options and the variety of pages dedicated to consumer education, observation, and data perusal.

The satellite images are cultural artifacts that represent a number of intersecting themes. The Environment Canada Website 
provides a historical social discourse whereby the satellite's mechanical gaze represents the colonizing "eye" of science dedicated to penetrating the mysterious veils of Mother Nature's mysterious cloak. Scientific classification and ordering of the atmosphere intersects with the conception of the weather as a mechanized entity subject to epistemic control, designed to facilitate the smooth running of social and economic systems. These different cultural interpretations of the weather illustrate how one perceives nature according to personal needs and productive desires. In an attempt to reintegrate sensual experience into the mechanized production of the weather, I explore the inversion of micro and macro perspectives whereby our gaze is displaced to an imaginary perspective in space through the portal of the computer screen.

Our perspective of the Earth's atmosphere has been colonized by modern science. It has become increasingly commonplace to understand weather in the context of mathematical systems and data sets. The science of meteorology has succeeded in classifying atmospheric phenomena and their flux in much the same way that scientists of the Enlightenment arranged and segregated the natural world into phylogenic categories and structures. For example, in 1967, meteorology pioneer Ted Fujita conducted the first of a series of Cloud Truth experiments (Menzel, 2001). Fujita's stereoscopic cloud tracking techniques opened the door to a new field of inferring atmospheric motions from remotely sensed image sequences. Fujita's early satellite meteorology reveals a tradition of rational scientific inquiry that characterizes weather experiments with satellite imagery. The whole notion of "cloud truth" reveals a preoccupation with the rational. The cloud, an intrinsically fluid, fluctuating atmospheric mass, is established as "real" and "fixed" through a process of visual reconnoitering and cross-examination, photographically freezing the cloud in time and space, and integrating it into a rational physical and temporal framework, in order to validate its existence and its role within a greater system of "true" things. This system of rational truths continues to expand as sophisticated sensing equipment estab- 
lishes and validates new patterns and flows in the ever-fluctuating atmosphere.

In the early stages of satellite meteorology, visual validity was confined to those parts of the light spectrum detectable by human eyes; infrared remote sensors have expanded the field of visual confirmation. The relationship between vision and truth is further mediated by, and in fact contingent upon, the technology that makes these spectral territories accessible to scientific discovery and intellectual colonization. It is no surprise that meteorologists were called pioneers; their explorations into the troposphere, while not physical in nature, represented an opening up of the mysterious, often epistemologically clouded sky to the penetrating gaze of science. The ordering and classification of weather patterns by means of sensory "eyes" is thus very much in line with Foucault's attention to the primacy of vision and its role in the origination of rational scientific inquiry and the development of natural laws (1970).

Satellite-assisted knowledge of the weather has pushed aside more traditional understandings of climate and seasonal change. Old adages like "Red sky at night, sailor's delight, red sky in the morning, sailors take warning," hold only a quaint nostalgia next to satellite colour-coded images. The truth-value of these sayings are contingent on the fact that hard science will validate such poesy, regardless of their ongoing, albeit low-tech utility. Another example involves the study of sparrows in the sky: "a low-flying sparrow brings a storm." Such an intimate, organic understanding of the weather involves insightful observations of one's immediate environment and the organisms within it. This intimacy of the old adages, while it may still exist nominally to some, takes second place to the hard facts of low-pressure systems and cloud refraction. These observations are also explicitly ground-based; the perspective is definitely from below, looking up into the sky. Modern meteorology sees the sky from far above, looking down God-like at the Earth. This perspective reasserts the dominance of Mankind over the atmospheric turmoil going on below and calls forth manifest destiny to continue the project 
of dominating the last frontier, not through coercion or force, but through the ordering, reasoning power of science.

This change in perspective is multi-faceted. Ironically, we look at a computer screen to understand what is happening right outside, or farther out in the troposphere. This reversal of the macro and micro results in a kind of sublimation of the self, whereby our perspective is transported to an imaginary space removed physically, temporally, and aesthetically from traditional, sensual experiences of nature and atmosphere. Our memories tell us that this digital synthesis is an image of the Earth's sky but our gaze is that of the satellite machine; the gaze of science witnessing phenomena previously inaccessible to our limited human sensory scope.

Satellite-mediated descriptions of the weather are reminiscent of Benjamin's inquiry into the nature of artistic authenticity in the age of mechanical reproduction (see "The Work of Art in the Age of Mechanical Reproduction.” Illuminations: Essays and Reflections, 1968), and based on his work inquiries into digital reproduction also now appear. The reproduction (if not direct simulation) of the Earth's atmosphere within the confines of a tiny computer screen entails a profound adjustment of reality for the viewer. The skies have a different meaning now that they are understood in a technological context. A synthetic display of data sets and invisible (infrared) light rendered into colour-coded patterns is not only a reproduction of the authentic sky, it is an elaboration of the visible original that includes previously unknown elements. This detailed technological elaboration is a sort of multi-dimensional unfolding that reveals the temporal under-painting of the work of nature that is the global climate; no longer a monument in its own right, but instead one instance, one factor, or one brush-stroke within a grand mathematical formula -- its microscopic and micro-temporal elements are revealed by the incisive gaze of remote sensing equipment. The digital view is a simulacrum of the atmosphere which obviously reveals more to the eye, on a grander scale, than a glance up at the original. 
A visually and temporally amplified sky, albeit engrossing, intellectually, and sometimes necessary for survival, via its stimulus and unnatural multiplicity; it misses out on the multi-sensual experience of the weather in its authentic form. Gone are the feelings of warmth, cool, dry, wet, or the sounds of wind and rain; the experience is uniquely visual. Understandings of any given weather systems are rational, remote, and purely intellectual; they exist independently of the experience of being inside that system, or even under it. Further, even if one wanted to experience the information in an authentic, sensual way, the physical limitations of one's earthly body would make that act impossible. Suppose a person was to go outside, and see that same cloud hanging in the sky that I witness above. He or she can feel the wind on his or her face, or feel if the air on the ground is mild. Yet, somehow, the infrared map superimposes itself in this experience of the real sky, and I now see this cloud simultaneously from below and above in a mental comparison; the experience becomes a set of measurements as much as a moving shape in the air. My perception adjusts to this new reality, and the skies are no longer mysterious and powerful; what I see and feel are simply phenomena within a matrix, a formula that will eventually account for, and predict, the movement of every molecule in the atmosphere.

Scientific narratives alone, however, cannot explain our culture's relationship with the weather, which is in a constant state of flux between animistic conceptions and satellite visions. We talk about the weather more often than we talk about sex, and indeed the two are culturally regulated in similar ways. As a natural phenomenon, indeed the natural phenomenon that makes Earth what it is in relation to neighbouring celestial bodies, weather suffuses all aspects of life and culture. It is the invisible hand of God to some, or even Zeus striking down the unworthy with bolts of lightning or Demeter blessing the fields with gentle rains and warm breezes to others, depending on the historical context and time period. Contemporary "storm porn" and Hollywood disaster movies have demonized inclement weather in much the same way. 
Our animistic understanding of the weather remains in contemporary society. "It" conspires to rain on the weekend only to clear up on Monday, playing a mean trick on productive workers who spend the week sealed in office buildings and are then denied the balmy weekend that is their right due. We do our best to avoid "bad" weather and hope for the kind of "good" weather that will facilitate the smooth running of social and economic systems. However, our everyday experience of the weather is rarely so dramatic as to conjure images of divine retribution or even divine indifference. For the most part, weather is a commonplace thing through which we must navigate in order to get on with economic labour and production.

Modern navigation through the atmosphere involves a synthesis of the mundane with a cyborg expansion of self into the extraterrestrial satellite-machine. We process these reconfigured images naturally, simultaneously in epistemic control, yet are still subject to the visceral effects of the wind and sun. Our engagement with new weather technologies is a new level of intimacy with the atmosphere that can synthesize one's direct sensual experiences with a greater scale of perspective to achieve a more profound understanding of the surrounding environment. That being said, it is often too easy to find one's self isolated on the synthetic side of things, sitting indoors staring trance-like into a computer screen while the clouds move on outside. This technological enframing may result, or has already resulted, in a reinforcement of the nature-human dualism whereby rational understanding supplants visceral understanding. The unmediated, sensual experience is thus conceived as threatening as it exists outside the safe, predictable and artificial realms of weather forecasting.

Where does this leave the casual, enchanted observer? The context within which we now experience these satellite images, the social, political and technological subplot, threatens to undermine the beauty of these new satellite images and the (perhaps naïve) wonder that they inspire. That satellite representations are part of a scientific and techno-colonial discourse does not negate the fact 
that such renderings have benefited society by means of preemptive storm forecasting and disaster relief. Nor should it disregard the undeniably practical and productive uses of modern weather forecasting for a modern sage or the fact that the weather was understood and functioned as a cultural phenomenon long before the advent of any official "weather science." However, value exists in examining and questioning the cultural meaning of this wealth of data, in all its visible and invisible forms, and how the nature of our evolving understanding of the weather will affect the future of our relationship with the Earth's atmosphere as a cultural product and as a phenomenon to be experienced sensually - with one's feet firmly planted on the ground.

\section{Works Cited}

Benjamin, Walter. "The Work of Art in the Age of Mechanical Reproduction.” Illuminations: Essays and Reflections. New York: Schocken Books, 1968.

Berland, Jody. "Weathering the North: Climate, Colonialism, and the Mediated Body." Relocating Cultural Studies: Developments in Theory and Research. Eds. Valda Blundell, John Shepherd and Ian Taylor. London: Routledge, 1993: 207-225.

Foucault, Michel. The Order of Things: An Archeology of the Human Sciences. New York: Vintage Books, 1970.

Gregory, Derek. “(Post)Colonialism and the Production of Nature.” Social Nature: Theory, Practice, Politics. Eds. Noel Castree \& Bruce Braun. Oxford: Blackwell, 2001. 84-110.

Menzel, Paul W. "Cloud Tracking with Satellite Imagery: From the Pioneering Work of Ted Fujita to the Present." Bulletin of the American Meteorological Society 82.1 (2001): 33-47. 